\title{
Zwei Psychotherapieverfahren im Vergleich
}

Fragestellung: Sind die psychodynamische Kurzzeittherapie und die kognitive Verhaltenstherapie bei der ambulanten Behandlung von Major Depression gleichwertig?

Hintergrund: Psychodynamische Therapien werden häufig angewandt, aber es gibt vergleichsweise wenige randomisierte klinische Studien, die deren Effektivität krankheitsspezifisch überprüft haben. In ambulanten Gesundheitszentren der USA, in denen viele Geringverdiener und Patienten ohne Krankenversicherung behandelt werden, sind Behandlungserfolge bei Major Depression im Allgemeinen geringer als bei klinischen Studien mit hoch selektionierten Patienten. Daher muss die Wirksamkeit von Therapieverfahren in diesem Setting separat geprüft werden.

Patienten und Methodik: Im Rahmen einer randomisierten kontrollierten Non-Inferiority-Studie erhielten Patienten mit Major Depression über fünf Monate verteilt jeweils 16 Sitzungen ambulante Psychotherapie, entweder als kognitive Verhaltenstherapie nach Beck oder als psychodynamische Psychotherapie nach Luborsky. Primäre Zielgröße war die Verbesserung der depressiven Symptomatik, gemessen mit der Hamilton Rating Scale for Depression (HAMD). Eingeschlossen

Connolly Gibbons MB, Gallop $\mathrm{R}$, Thompson D et al. Comparative effectiveness of cognitive therapy and dynamic psychotherapy for major depressive disorder in a community mental health setting. A randomized clinical noninferiority trial. JAMA Psychiatry 2016; 73: 904-11 wurden 18 - bis 65 -jährige Ratsuchende eines ambulanten Gesundheitszentrums in den USA, die um die Behandlung ihrer depressiven Symptomatik gebeten hatten, Werte von > 10 im Quick Inventory for Depressive Symptomatology aufwiesen und in einem telefonischen Screening hinsichtlich psychopathologischer Symptome auffällig waren. Diese Patienten wurden anschließend mit dem Strukturierten Klinischen Interview für DSM-IV (SKID, Achse I) durch geschulte, externe Begutachter untersucht. Die Randomisation erfolgte stratifiziert durch einen bei der Behandlung nicht involvierten Statistiker. Die Adhärenz der Therapeuten zum jeweiligen Therapieverfahren wurde anhand von Fragebögen durch die Patienten beurteilt. Die statistische Analyse erfolgt anhand hierarchischer linearer Regressionsmodelle, wobei a priori ein Nicht-Unterlegenheitsbereich von 2,5 Punkten festgelegt worden war.

Ergebnisse: 237 Patienten wurden randomisiert zu ambulanter psychodynamischer $(n=118)$ oder kognitiver Therapie $(n=119)$ zugeteilt und engmaschig supervidiert. Die Gruppen waren hinsichtlich wichtiger soziodemografischer Merkmale vergleichbar, allerdings waren in der Gruppe mit kognitiver Therapie mehr Patienten mit höherer Bildung. 103 (87\%) der Patienten bei psychodynamischer und 99 (83\%) bei kognitiver Therapie nahmen mindestens eine Behandlungsstunde in Anspruch. Die Veränderung der depressiven Symptomatik in beiden Gruppen lag mit 0,86 Punkten Unterschied zwischen den beiden Therapieverfahren innerhalb des Nicht-Unterlegenheitsbereichs, die obere Grenze des $95 \%$-Konfidenzintervalls betrug 2,4. Medikation wirkte dabei nicht effektmodifizierend. In beiden Verfahren gab es eine statistisch signifikante Verbesserung der Symptomatik über die Zeit.

Schlussfolgerungen: Bei der Behandlung von Major Depression im ambulanten Setting ist psychodynamische Therapie einer kognitiven Therapie hinsichtlich der Besserung der depressiven Symptomatik nicht unterlegen.

\section{- Kommentar von Susanne Singer, Mainz}

\section{Bei der Therapiewahl den Patientenwunsch mit berücksichtigen}

Diese Studie überzeugt dadurch, dass sie einerseits sehr sorgfältig durchgeführt wurde, andererseits dem realen klinischen Alltag entspricht. So ist es zum Beispiel positiv zu werten, dass Patienten mit komorbiden Angsterkrankungen nicht ausgeschlossen wurden. Insgesamt hat die Studie damit eine hohe externe Validität. Bei Non-Inferiority-Studien müssen einige Besonderheiten in der Methodik beachtet werden, beispielsweise muss die Fallzahl höher sein. Dies wurde von den Autoren berücksichtigt. Insgesamt wurden alle Anforderungen an Durchführung und Berichterstattung bei randomisierten klinischen Studien sehr gut erfüllt, sofern sie bei der Beantwortung der Fragestellung machbar waren. Die Studie war nicht verblindet, dies ist aber bei Psychotherapiestudien auch nicht möglich. Insgesamt werden vier von fünf Jadad-Punkten erreicht, das Evidenzniveau nach der Cochrane-Klassifikation liegt bei Ib. Die Ergebnisse zeigen, dass sowohl kognitive Ver- haltenstherapie als auch psychodynamische Psychotherapie in der ambulanten psychotherapeutischen Behandlung von Patienten mit Major Depression wirksam sind. Bei der Indikation für ein bestimmtes Therapieverfahren kann und sollte daher die Präferenz des Patienten mit berücksichtigt werden.

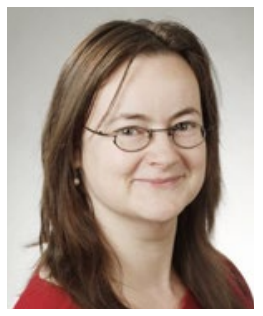

Prof. Dr. Susanne Singer, Mainz

Klinik für Psychiatrie und Psychotherapie sowie Institut für Medizinische Biometrie, Epidemiologie und Information, Abt. Epidemiologie und Versorgungsforschung, Universitätsmedizin Mainz E-Mail: susanne.singer@unimedizin-mainz.de 